\title{
The antitumor natural compound falcarindiol promotes cancer cell death by inducing endoplasmic reticulum stress
}

\author{
HR Jin ${ }^{1}$, J Zhao ${ }^{1}$, Z Zhang ${ }^{2,3}$, Y Liao ${ }^{1}$, C-Z Wang ${ }^{2,3}$, W-H Huang ${ }^{4}$, S-P Li' ${ }^{4}$ T-C He ${ }^{5}$, C-S Yuan ${ }^{2,3}$ and W Du ${ }^{*, 1}$
}

Falcarindiol (FAD) is a natural polyyne with various beneficial biological activities. We show here that FAD preferentially kills colon cancer cells but not normal colon epithelial cells. Furthermore, FAD inhibits tumor growth in a xenograft tumor model and exhibits strong synergistic killing of cancer cells with 5-fluorouracil, an approved cancer chemotherapeutic drug. We demonstrate that FAD-induced cell death is mediated by induction of endoplasmic reticulum (ER) stress and activation of the unfolded protein response (UPR). Decreasing the level of ER stress, either by overexpressing the ER chaperone protein glucoseregulated protein 78 (GRP78) or by knockout of components of the UPR pathway, reduces FAD-induced apoptosis. In contrast, increasing the level of ER stress by knocking down GRP78 potentiates FAD-induced apoptosis. Finally, FAD-induced ER stress and apoptosis is correlated with the accumulation of ubiquitinated proteins, suggesting that FAD functions at least in part by interfering with proteasome function, leading to the accumulation of unfolded protein and induction of ER stress. Consistent with this, inhibition of protein synthesis by cycloheximide significantly decreases the accumulation of ubiquitinated proteins and blocks FAD-induced ER stress and cell death. Taken together, our study shows that FAD is a potential new anticancer agent that exerts its activity through inducing ER stress and apoptosis.

Cell Death and Disease (2012) 3, e376; doi:10.1038/cddis.2012.122; published online 23 August 2012

Subject Category: Cancer

Cancer is a leading cause of death and colorectal cancer is the second leading cause of total cancer deaths in the United States. ${ }^{1}$ Current treatment of colorectal cancer generally employs surgical resection combined with radiation therapy and chemotherapy using one or more cytotoxic drugs. This therapy is only moderately successful for late-stage cancers and is often limited by severe side effects and dose-limiting toxicity. ${ }^{2,3}$ Therefore, identifying new, less toxic chemoadjuvants from herbal medicine that can selectively kill cancer cells or enhance the effects of existing chemotherapeutic agents can potentially lead to the development of better treatment for late-stage colorectal cancers.

Cancer cells exhibit hallmarks of increased cellular stresses including DNA damage/replication stress, proteotoxic stress, mitotic stress, metabolic stress, and oxidative stress, ${ }^{4}$ and are more dependent on stress support pathways for survival. The increased dependence of cancer cells on these pathways for survival can be exploited in cancer therapy by either stress sensitization or stress overload. In fact, currently used and effective cancer therapies, such as radiation and DNA damaging agents, potentially kill cancer cells through inducing stress overload. ${ }^{4}$
Endoplasmic reticulum (ER) is the site for proper protein folding of secreted and transmembrane proteins. Accumulation of unfolded or misfolded proteins in the ER will induce ER stress and trigger an evolutionarily conserved response called the unfolded protein response (UPR). ${ }^{5,6}$ Three distinct branches of the UPR have been identified based on distinct sensors: IRE1, PKR-like endoplasmic reticulum kinase (PERK), and ATF6. Activation of IRE1 induces X-box binding protein 1 (XBP1) splicing to generate the active form of the XBP1 transcription factor. Activation of PERK leads to phosphorylation of elF2 $\alpha$, which inhibits global translation of mRNAs and reduces the influx of new proteins into the ER to mitigate the burden on the ER protein folding machinery. However, the translation of a subset of mRNAs, such as ATF4, is upregulated. Activation of the transcription factors XBP1 and ATF4, as well as ATF6 by the third branch of UPR, will activate UPR target genes including ER chaperones, ERassociated protein degradation (ERAD) genes, genes involved in autophagy, and genes involved in apoptosis. ${ }^{5,6}$ Therefore, UPR signaling activates programs that promote ER homeostasis and survival, as well as programs that can induce cell death.

\footnotetext{
${ }^{1}$ Ben May Department for Cancer Research, The University of Chicago, Chicago, IL, USA; ${ }^{2}$ Tang Center for Herbal Medicine Research, The University of Chicago, Chicago, IL, USA; ${ }^{3}$ Department of Anesthesia and Critical Care, The University of Chicago, Chicago, IL, USA; ${ }^{4}$ State Key Laboratory of Quality Research in Chinese Medicine, and Institute of Chinese Medical Sciences, University of Macau, Macao, China and ${ }^{5}$ Department of Surgery, The University of Chicago, Chicago, IL, USA ${ }^{*}$ Corresponding author: W Du, Ben May Department for Cancer Research, The University of Chicago, 929 East 57th Street, Chicago, IL 60637, USA. Tel: +773 834 1949; Fax: +773 702 4476; E-mail: wei@uchicago.edu

Keywords: ER stress; falcarindiol; apoptosis; unfolded protein response; proteasome

Abbreviations: ER, endoplasmic reticulum; UPR, unfolded protein response; GRP78, glucose-regulated protein 78; XBP1, X-box binding protein 1; CHOP, C/EBPhomologous protein; PERK, PKR-like endoplasmic reticulum kinase; ERAD, ER-associated protein degradation; LC3, microtubule-associated protein light chain 3 Received 25.4.12; revised 6.7.12; accepted 19.7.12; Edited by A Finazzi-Agro'
} 
The main targets of UPR signaling that promote survival include the ER chaperone glucose-regulated protein 78 (GRP78) and ERAD genes. GRP78 is required for proper protein folding and assembly in the ER, targeting misfolded proteins for degradation, $\mathrm{ER} \mathrm{Ca}^{2+}$ binding, and controlling the activation of transmembrane ER stress receptors. GRP78 is often upregulated in cancer cells and is potentially a target for cancer therapy. ${ }^{7}$ On the other hand, terminally misfolded proteins are removed by the ERAD machinery through ubiquitin-mediated protein degradation, which helps maintain ER homeostasis and cell survival. ${ }^{8}$ If the function of the ER cannot be reestablished by UPR, excessive or sustained ER stress will induce cell death via IRE1-mediated activation of JNK signaling as well as through prolonged activation of the transcription factor C/EBP-homologous protein (CHOP). ${ }^{9}$ BCL2 family proteins are implicated in $\mathrm{CHOP}$-induced apoptosis. In addition, BCL2 family of proteins are also critically linked to cell life and death through modulating ER calcium homeostasis. ${ }^{10}$ The cell death mechanisms triggered by ER stress include both caspase-dependent apoptosis and caspase-independent necrosis. ${ }^{11}$

Falcarindiol (FAD) is a natural polyyne that has been shown to have anti-inflammation, ${ }^{12,13}$ antibacterial, ${ }^{14,15}$ and anticancer $^{16}$ activities, as well as protective effects against hepatotoxicity. ${ }^{17}$ Moreover, these beneficial effects occur at non-toxic concentrations and thus represent pharmacologically useful properties. In this report, we show that FAD preferentially kills colorectal cancer cells and inhibits tumor growth, which is mediated by the induction of ER stress as a consequence of inhibiting proteasome function and causing accumulation of ubiquitinated proteins. Furthermore, FAD exhibits a strong synergistic effect in killing cancer cells in conjunction with 5-fluorouracil (5-FU), an approved chemotherapeutic drug for colorectal cancer. These results suggest that FAD can potentially be used to develop better and safer new therapies for colorectal cancers.

\section{Results}

FAD preferentially induces human cancer cell death. To determine whether FAD can preferentially kill cancer cells, we compared the ability of FAD to induce cell death in HCT116 and SW480 human colorectal cancer cells with that in FHC normal human colon epithelial cells. As shown in Figures 1a and b, FAD kills both cancer cells in a dosedependent manner and a low micromolar concentration of $F A D$ is sufficient to induce significant levels of cell death in both cases. In contrast, FAD treatment does not induce a significant level of cell death in FHC normal human colon epithelial cells at 5-10 $\mu \mathrm{M}$ concentration (Figure 1c). In fact, the majority of FHC cells can survive $20 \mu \mathrm{M}$ of FAD, even though a statistically significant level of cell death is observed at this concentration (Figure 1c). To further determine whether FAD can kill different types of cancer cells, we tested the effects of FAD on several cancer cells derived from different tissues (MDA-MB231, MCF7, DU145, MonoMac6, Raji, and KOPN-1) as well as non-transformed MCF10A and IEC6 cells. Although non-transformed cells are resistant to FAD treatment, most of the cancer cells, with the exception of MCF7, are sensitive to $6 \mu \mathrm{M}$ of FAD
(Supplementary Figure S1). These data indicate that FAD can preferentially kill cancer cells and that significant numbers of human cancers are sensitive to FAD.

FAD induces caspase-dependent apoptotic cancer cell death. Three types of cell death are commonly induced in cancer cells: apoptosis that is mediated by the activation of caspases; necrosis that does not require caspase activity; and autophagic cell death. Recent studies show that necroptosis, a form of cell death that is regulated but exhibits the same morphological features as unregulated necrotic death, can be specifically inhibited by necrostatins. ${ }^{18}$ To determine whether apoptosis contributes to FAD-induced cell death in cancer cells, we stained HCT116 cells with Annexin $\mathrm{V}$. FAD treatment induces a significant increase in Annexin $\mathrm{V}$ staining (Figures 1d and e), suggesting that significant levels of apoptosis are induced by FAD. In support of this hypothesis, the pan-caspase inhibitor Z-VAD-fmk significantly inhibits FAD-induced cell death $(P<0.01$, Figure 1f) whereas the necroptosis inhibitor Necrostatin-1 does not $(P=0.25$, Figure 1g). Therefore, FAD-induced cancer cell death is mediated mostly by caspase-dependent apoptosis.

The microtubule-associated light chain 3 (LC3) is cleaved upon autophagy induction to form the faster migrating LC3-II form, which is conjugated to phospholipids and incorporated into the autophagosomes. Both the conversion of LC3-I to LC3-II and the formation of foci by GFP-tagged LC3 (GFPLC3) are often used as markers for autophagy induction. ${ }^{19}$ To determine whether autophagy contributes to FAD-induced cancer cell death, we examined the effect of FAD on the induction of LC3-II. As shown in Figure 1i, significant levels of LC3-I are converted to LC3-II at $24 \mathrm{~h}$ after FAD treatment. In addition, significantly increased numbers of GFP-LC3 foci are observed after HCT116 cells were treated with $3 \mu \mathrm{M}$ FAD as compared with the vehicle control (Figure 1j), indicating autophagy is induced by FAD treatment.

To determine whether increased level of autophagy contributes to FAD-induced cell death, we examined the effect of autophagy inhibition by chloroquine. As shown in Figure $1 \mathrm{~h}$, while $3 \mu \mathrm{M}$ of $\mathrm{FAD}$ on its own can induce a significant level of autophagy as shown by increased GFPLC3 foci formation (Figure 1j) and a low level of cell death (Figure 1h), blocking autophagy with chloroquine exacerbated FAD-induced cell death $(P<0.05$, Figure $1 \mathrm{~h})$. These data indicate that the increased autophagy observed upon FAD treatment does not contribute to FAD-induced cell death, but rather appears to have a protective role limiting the extent of FAD-induced cell death.

FAD induces ER stress in cancer cells. Autophagy can be induced by a variety of cellular stresses and has a cytoprotective role in chemotherapeutic treatment by removing excess cellular stress. ${ }^{20-22}$ To determine whether apoptosis induced by FAD is correlated with ER stress induction, we investigated whether FAD treatment could induce GRP78 and CHOP expression and XBP1 splicing. As shown in Figure 2, FAD increases GRP78 protein in a doseand time-dependent manner (Figures $2 a$ and $b$ ). Furthermore, FAD also induces XBP1 splicing and CHOP mRNA accumulation in a dose-dependent manner (Figure 2c). 
a

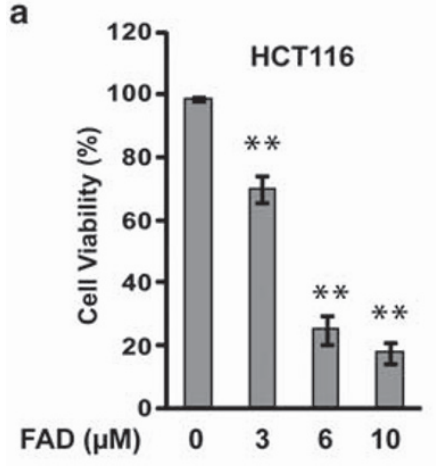

b

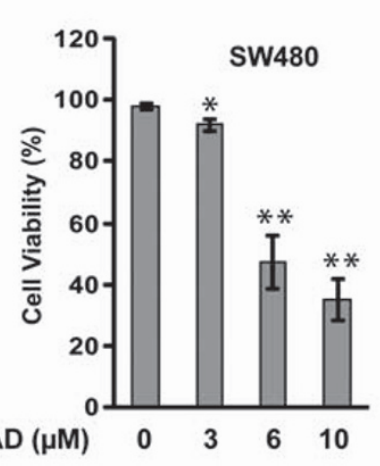

c

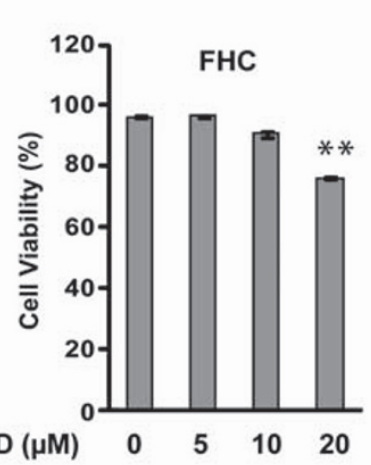

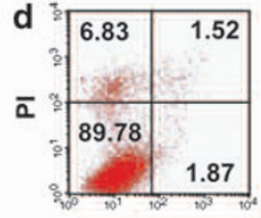

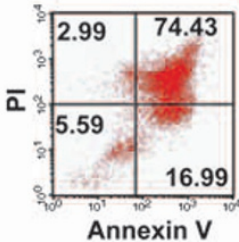

e

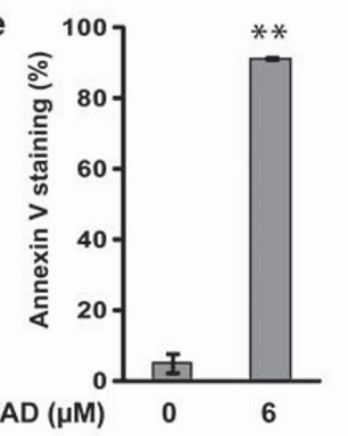

f

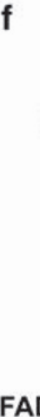

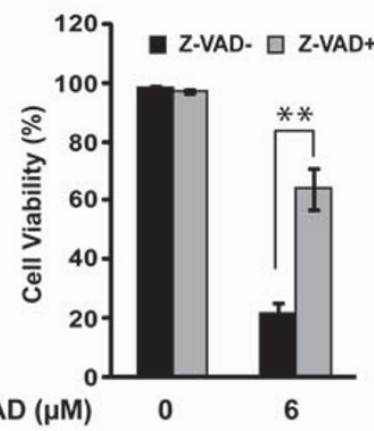

g
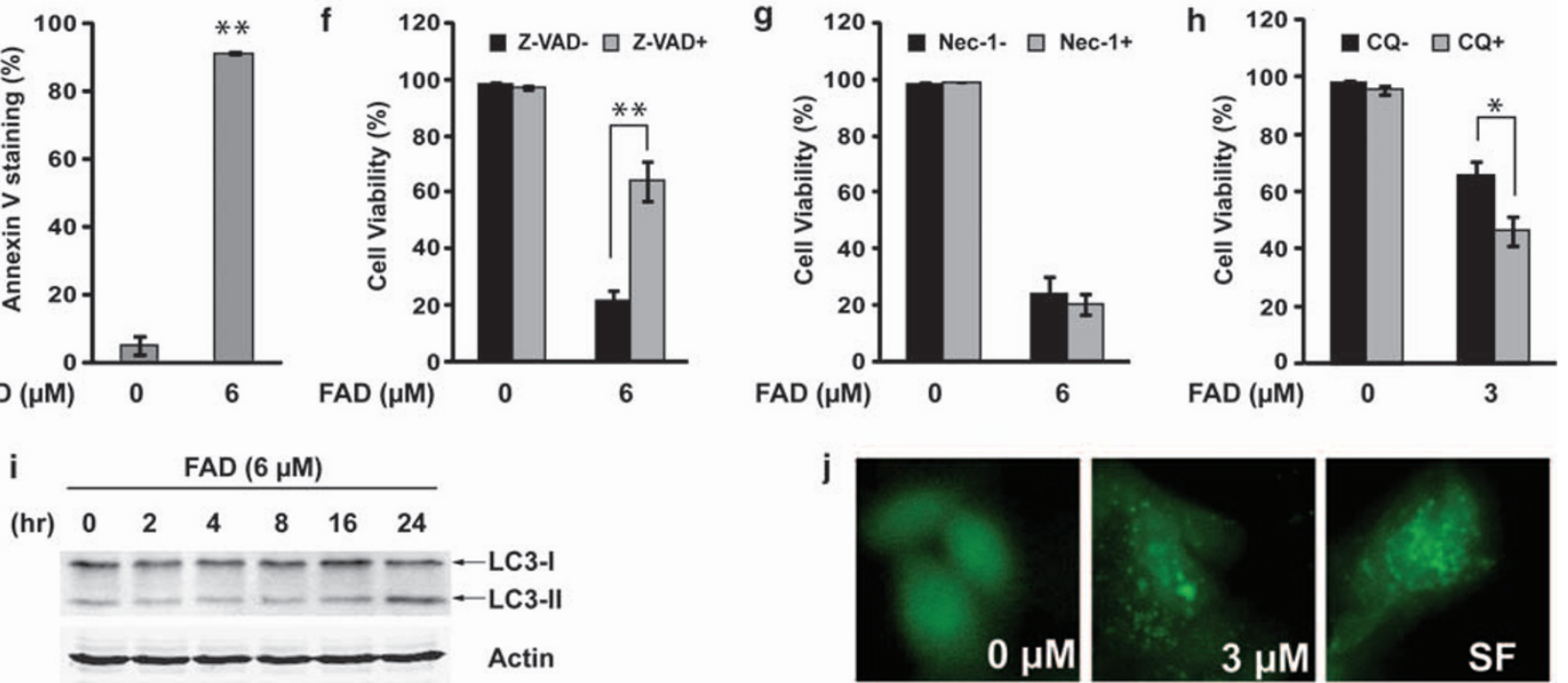

j

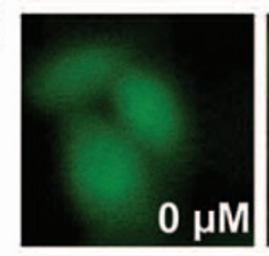

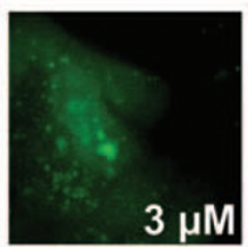

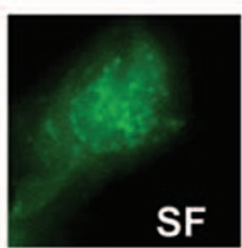

Figure 1 The effects of FAD on colorectal cancer cell lines. (a and $\mathbf{b})$ Human colorectal cancer cells HCT116 and SW480 were treated with different concentrations of FAD for $48 \mathrm{~h}$ and cell viability was quantified. (c) Normal human colon epithelial cells FHC were treated with different concentrations of FAD for $48 \mathrm{~h}$ and cell viability was quantified. (d and e) HCT116 cells were treated with FAD for $48 \mathrm{~h}$, and apoptosis was quantified by FACS after staining with Annexin V-FITC and propidium iodide. (f) Viability of HCT116 cells treated with $6 \mu \mathrm{M}$ FAD for $48 \mathrm{~h}$ in the presence or absence of $100 \mu \mathrm{M}$ Z-VAD-fmk. (g) Viability of HCT116 cells treated with $6 \mu \mathrm{M} \mathrm{FAD} \mathrm{for} 48 \mathrm{~h}$ in the presence or absence of $40 \mu \mathrm{M}$ Necrostatin-1 (Nec-1). (h) Viability of HCT116 cells treated with $3 \mu \mathrm{M}$ FAD for $48 \mathrm{~h}$ in the presence or absence $20 \mu \mathrm{M}$ of chloroquine (CQ). Trypan blue staining was used to determine cell viability. (i) HCT116 cells were treated with $6 \mu \mathrm{M} \mathrm{FAD} \mathrm{for} \mathrm{the} \mathrm{indicated} \mathrm{periods,} \mathrm{and} \mathrm{LC3-I/II} \mathrm{protein} \mathrm{levels} \mathrm{were} \mathrm{determined} \mathrm{by} \mathrm{western} \mathrm{blot} \mathrm{analysis.} \mathrm{(j)}$ HCT116 cells expressing EGFP-LC3 were treated with 0 or $3 \mu \mathrm{M}$ FAD for $30 \mathrm{~h}$ and were imaged with a fluorescence microscope. Serum-free (SF) treatment was used as a positive control for autophagy induction. ${ }^{*} P<0.05$ and ${ }^{\star \star} P<0.01$

Interestingly, FAD-induced XBP1 splicing and CHOP message are detectable as early as $2 \mathrm{~h}$ and nearly maximal levels of XBP1 splicing and CHOP mRNA are observed within $8 \mathrm{~h}$ of FAD treatment (Figure 2d). In contrast, significantly increased GRP78 protein and message levels are observed after $8 \mathrm{~h}$ of $F A D$ treatment (Figures $2 \mathrm{~b}$ and $\mathrm{d}$ ), significantly later than the induction of $\mathrm{CHOP}$ expression and XBP1 splicing. As CHOP functions to promote ER stress-induced cell death whereas GRP78 protects cells from ER stressinduced cell death, the rapid induction of proapoptotic genes such as CHOP and the relatively slow induction of protective mechanisms such as GRP78 by FAD treatment potentially explain cell death induction in HCT116 cells.

To determine whether ER stress induction correlates with FAD-induced cell death, we determined the level of XBP1 splicing. As shown in Supplementary Figure S2, cell lines that are sensitive to FAD exhibit high level of XBP1 splicing after $F A D$ treatment whereas cell lines that are resistant to $F A D$ exhibit little FAD-induced XBP1 splicing (compare Supplementary Figures S1 and S2a). Therefore, the ability of $F A D$ to induce ER stress correlates with FAD-induced cell death.

FAD-induced apoptosis is mediated by ER stress. If FAD-induced cell death is mediated by ER stress, treatment that leads to decreased ER stress is expected to decrease FAD-induced cell death whereas treatment that increases ER stress should enhance FAD-induced cell death. As the ER chaperone GRP78 functions as an inhibitor of ER stress receptors and inhibits ER stress-mediated cell death, we determined the effects of either GRP78 overexpression or knockdown on FAD-induced cell death. Overexpression of GRP78 was achieved by infecting HCT116 cells with lentivirus that expresses a myc-tagged GRP78 construct (myc-GRP78, Figure 3b). In support of the hypothesis that FAD-induced cell death is mediated by ER stress, expression 
of GRP78 significantly attenuated FAD-induced cell death ( $P<0.05$, Figure 3a). Furthermore, the reduced cell death is correlated with significantly decreased levels of ER stress as
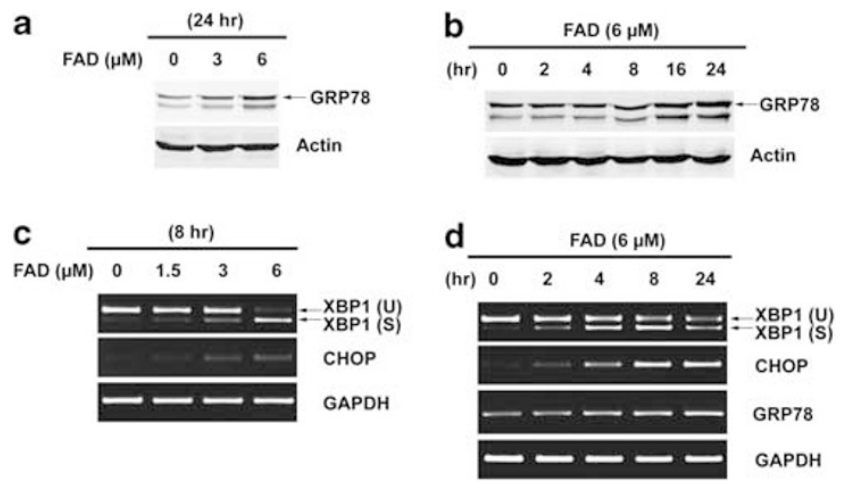

Figure 2 FAD induces endoplasmic reticulum stress in colorectal cancer cells. (a) Western blot showing the induction of GRP78 in HCT116 cells treated with different concentrations of FAD. (b) Western blot showing the induction of GRP78 in HCT116 cells treated with FAD at different time points. Full-length GRP78 protein is indicated by an arrow. (c) RT-PCR analysis showing the induction of XBP1 splicing and $\mathrm{CHOP}$ expression after $\mathrm{HCT} 116$ cells were treated with different concentrations of FAD. (d) RT-PCR analysis of indicated genes at different time points after HCT116 cells were treated FAD detected by decreases in XBP1 splicing and CHOP induction (Figure 3c).

shRNA-mediated GRP78 knockdown has been shown to sensitize cells to ER stress-induced cell death. ${ }^{23,24}$ To determine whether reducing the level of GRP78 can sensitize cells to FAD treatment, we infected HCT116 cells with lentivirus that expresses two different shRNAs that specifically knock down GRP78. Reduced GRP78 protein levels are detected in the sh-GRP78-knockdown cells (Figures 3d and e), which is correlated with significantly increased sensitivity to FAD-induced cell death $(P<0.01$, Figures $3 f$ and $\mathrm{g})$. Furthermore, the increased sensitivity of GRP78-knockdown cells to FAD-induced cell death is correlated with increased XBP1 splicing and CHOP induction (Figure 3h). These results provide strong evidence that FAD-induced cell death is mediated by ER stress induction.

FAD induces intracellular accumulation of ubiquitinated proteins in cancer cells. To further characterize the mechanism by which FAD induces ER stress and cancer cell death, we determined the effect of blocking protein synthesis by cycloheximide (CHX) on FAD-induced cell death. Inhibition of protein synthesis blocks the accumulation of unfolded/misfolded proteins in ER and therefore can potentially alleviate ER stress-induced cell death. ${ }^{25,26}$ As a

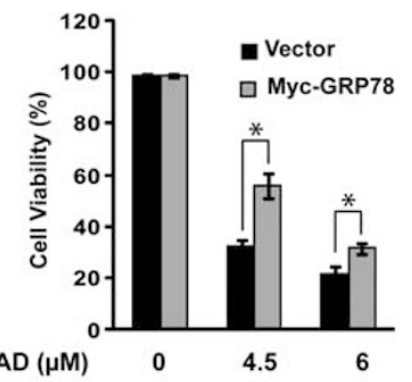

d

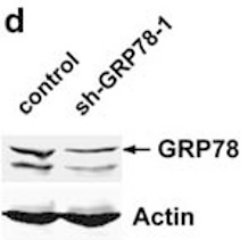

e
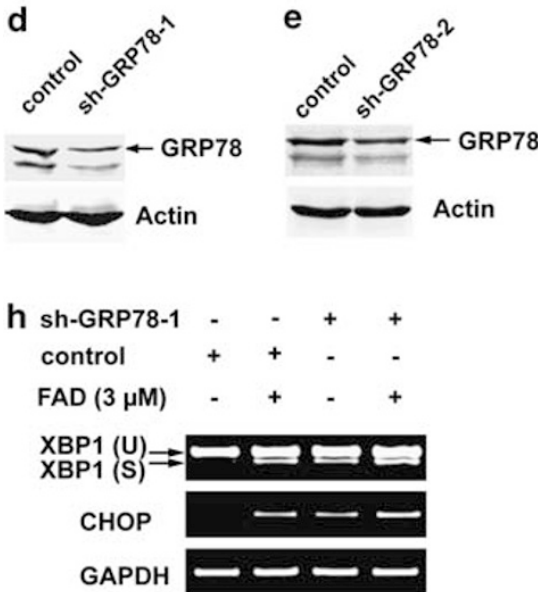

b

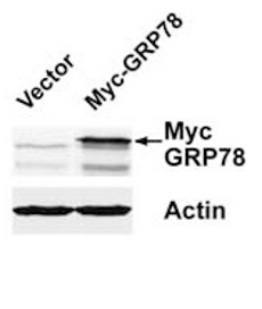

C

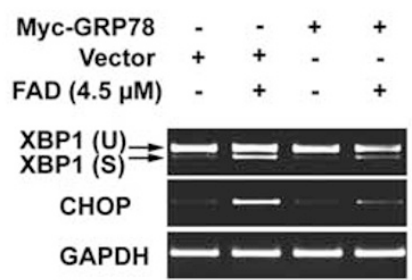

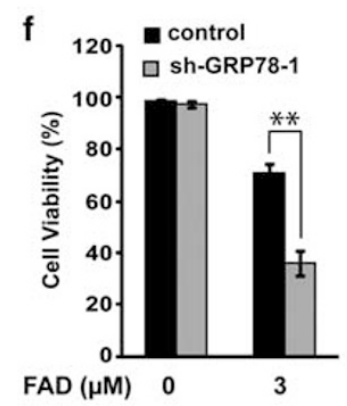

g

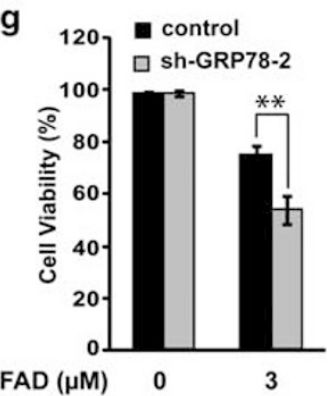

Figure 3 FAD induces ER stress-mediated apoptosis in colorectal cancer cells. (a) HCT116 cells infected with lentivirus expressing either myc-GRP78 or empty vector were treated with different concentrations of FAD for $48 \mathrm{~h}$, and cell viability was determined. (b) Extracts from vector control or myc-GRP78-expressing cells were analyzed by western blot. Anti-Myc antibody was used to detect myc-GRP78 (indicated by an arrow). The blot was reprobed with an anti-GRP78 antibody, which detects the endogenous GRP78 bands (below myc-GRP78). (c) RT-PCR analysis of the indicated genes after myc-GRP78 or vector control-infected HCT116 cells were treated with $4.5 \mu \mathrm{M}$ FAD for $8 \mathrm{~h}$. (d and $\mathbf{e}$ ) Extracts from vector control or sh-GRP78 cells were analyzed by western blots to determine the levels of GRP78 protein (indicated by arrow). (f and $\mathbf{g}$ ) HCT116 cells infected with different sh-GRP78 constructs or vector control were treated with $3 \mu \mathrm{M}$ FAD for $48 \mathrm{~h}$, and cell viability was determined. (h) RT-PCR analysis of the indicated genes after sh-GRP78 or control vector-infected HCT116 cells treated with $3 \mu \mathrm{M}$ FAD for $8 \mathrm{~h} .{ }^{*} P<0.05$ and ${ }^{* \star} P<0.01$ 


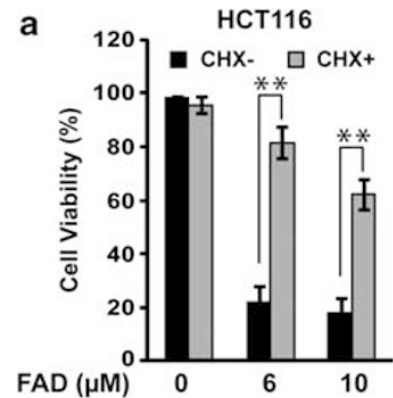

e
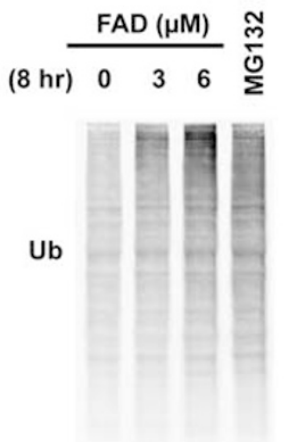

Actin $-\infty$ b

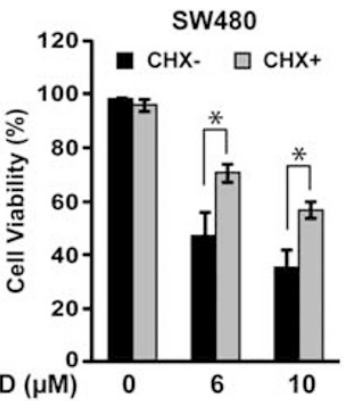

f

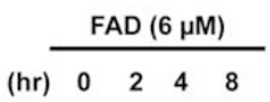

Ub

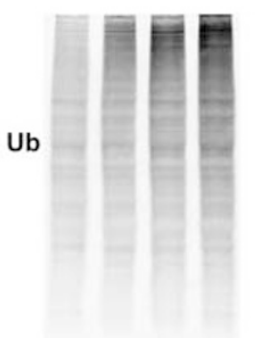

Actin c

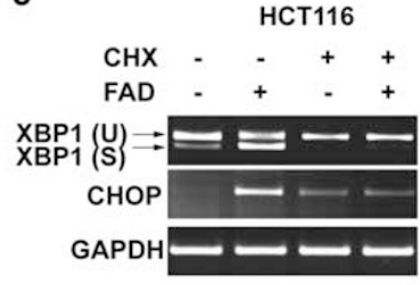

d

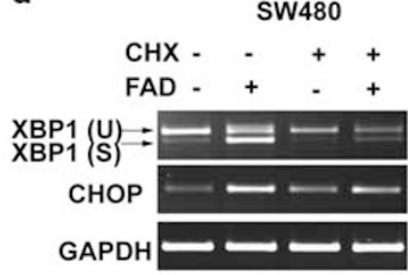

g

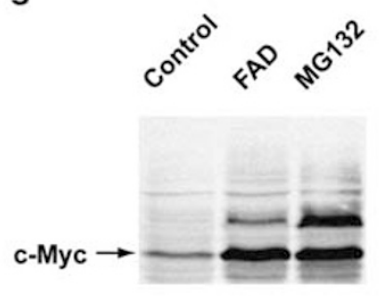

Actin $\rightarrow \geq$ * h

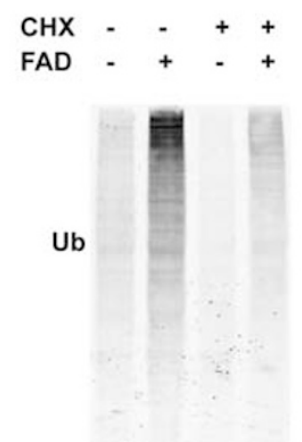

Actin - - -

Figure 4 FAD induces accumulation of ubiquitinated proteins in colorectal cancer cells. (a and b) HCT116 and SW480 cells were treated with the indicated concentrations of FAD for $48 \mathrm{~h}$ with or without cycloheximide $(10 \mu \mathrm{g} / \mathrm{ml})$, and cell viability was quantified. (c and d) HCT116 and SW480 cells were treated with $6 \mu \mathrm{M} \mathrm{FAD} \mathrm{for} 8 \mathrm{~h}$ with or without cycloheximide $(10 \mu \mathrm{g} / \mathrm{ml})$, and total RNA isolated from the cells was subjected to RT-PCR analysis with specific primers as indicated. (e) HCT116 cells were treated with indicated concentrations of $\mathrm{FAD}$, or $20 \mu \mathrm{M}$ proteasome inhibitor MG132 for $8 \mathrm{~h}$, and the levels of ubiquitinated proteins were determined by western blot analysis. (f) $\mathrm{HCT} 116$ cells were treated with $6 \mu \mathrm{M} \mathrm{FAD}$ for the indicated periods, and the levels of ubiquitinated proteins were determined by western blot analysis. (g) HCT116 cells were treated with $6 \mu \mathrm{M} \mathrm{FAD}$ or $20 \mu \mathrm{M}$ of MG132 for $8 \mathrm{~h}$, and the c-Myc protein level was determined by western blot analysis. (h) HCT116 cells were treated with $6 \mu \mathrm{M}$ FAD for $8 \mathrm{~h}$ in the presence or absence cycloheximide $(10 \mu \mathrm{g} / \mathrm{ml})$, and the level of ubiquitinated proteins were determined by western blot analysis. ${ }^{*} P<0.05$ and ${ }^{* *} P<0.01$

shown in Figures $4 a$ and $b$, pre-treatment with $\mathrm{CHX}$ significantly inhibits FAD-induced cell death. Furthermore, we found that cell death inhibition by $\mathrm{CHX}$ is correlated with an inhibition of FAD-induced XBP1 splicing and $\mathrm{CHOP}$ expression (Figures $4 \mathrm{C}$ and $\mathrm{d}$ ). These data further support the hypothesis that FAD-induced cell death is mediated by ER stress-induced apoptosis.

Terminally misfolded proteins in the ER are first removed from the ER via delivery to the cytosol, where they are degraded by the ubiquitin-proteasome system, a process commonly referred to as ER-associated degradation (ERAD). ${ }^{6}$ Indeed proteasome inhibitors have been shown to induce the accumulation of ubiquitinated proteins, ER stress, and apoptosis. ${ }^{27,28}$ To determine whether FAD-induced ER stress is correlated with an accumulation of ubiquitinated proteins, we used an anti-ubiquitin antibody. A dose-dependent increase in the level of ubiquitinated proteins is observed with increasing doses of $F A D$, similar to the effect of the proteasome inhibitor MG132 (Figure 4e). The accumulation of ubiquitinated proteins is rapid after FAD treatment, detectable as early as $2 \mathrm{~h}$ after FAD treatment (Figure $4 \mathrm{f}$ ). In addition, the accumulation of ubiquitinated proteins is observed in FADsensitive MDA-MB231 breast cancer cells but not in the FADresistant non-transformed $M C F 10 A$ breast epithelial cells (Supplementary Figure S2b). To further investigate whether FAD might interfere with proteasome-mediated protein degradation, we determined the level of c-Myc protein, which is an unstable protein degraded by the ubiquitin-proteasome pathway. ${ }^{29}$ Inhibition of the proteasome by MG132 leads to the stabilization of $\mathrm{c}-\mathrm{Myc}$ as well as the accumulation of high MW forms of c-Myc (Figure 4g), similar to the reported ubiquitination of c-Myc. ${ }^{29}$ Importantly, FAD treatment leads to a similar effect (Figure 4g), suggesting that FAD functions at least in part by interfering with proteasome function. Furthermore, inhibition of protein synthesis by $\mathrm{CHX}$ significantly reduces the accumulation of $F A D$-induced protein ubiquitination (Figure 4h), which is correlated with its inhibition of FADinduced ER stress (Figures $4 \mathrm{c}$ and $\mathrm{d})$ and cell death $(P<0.01$ or 0.05 , Figures $4 a$ and $b$ ).

Mouse embryonic fibroblasts with mutations in ER stress pathway components are more resistant to FADinduced cell death. To further confirm that FAD-induced cell death is associated with ER stress, we examined the levels of cell death and ER stress induced by FAD in WT control mouse embryonic fibroblasts (MEFs) or MEFs with homozygous deletion of either PERK or XBP1. Deletion of the PERK gene is reflected by the absence of PERK protein on western blots (Figure $5 \mathrm{~b}$ ). In support of the hypothesis that FAD-induced cell death is mediated by excessive ER stress, PERK ${ }^{-1-}$ MEFs exhibited decreased levels of FADinduced cell death $(P<0.05$, Figure 5a), which is correlated 


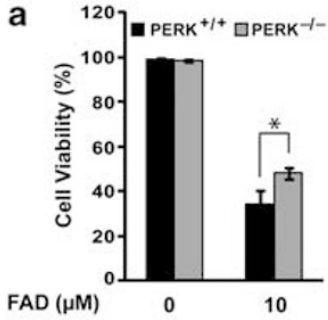

b

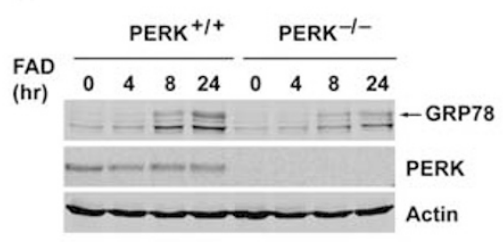

e

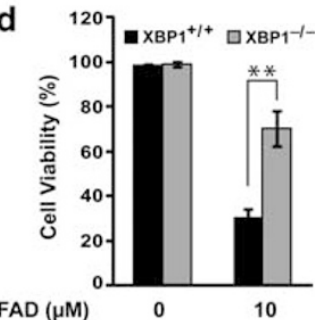

C

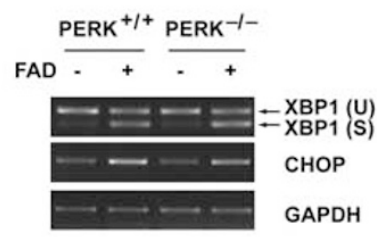

f

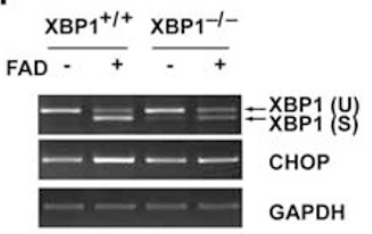

Figure 5 FAD-induced MEF cell death through induction of ER stress. (a and d) Wild-type and $P E R K^{-/}$or $X B P 1^{-/-}$mouse embryonic fibroblasts were treated with $10 \mu \mathrm{M} \mathrm{FAD}$ for $48 \mathrm{~h}$, and the cell viability was quantified. (b and $\mathbf{e})$ Wild-type and $P E R K^{-1-}$ or $X B P 1^{-1-}$ mouse embryonic fibroblasts were treated with $10 \mu \mathrm{M} \mathrm{FAD} \mathrm{for} \mathrm{the}$ indicated periods, and GRP78 and PERK expression levels were determined by western blot analysis. (c and f) Wild-type and PERK ${ }^{-1-}$ or XBP1 ${ }^{-1-}$ mouse embryonic fibroblasts were treated with $10 \mu \mathrm{M}$ FAD for $8 \mathrm{~h}$, and total RNA isolated from the cells was subjected to RT-PCR analysis with specific primers as indicated. Note: XBP1 knockout does not remove the region that is used to assay alternative splicing, therefore XBP1 splicing can still be detected in the XBP1 knockout MEF and used as a readout for ER stress. ${ }^{*} P<0.05$ and ${ }^{* *} P<0.01$

with decreased ER stress as shown by reduced levels of both CHOP and GRP78 (Figures 5c and b). In contrast, XBP1 splicing, which is mediated by IRE1 independent of PERK, is not affected (Figure 5c). Interestingly, XBP1 ${ }^{-1}$ MEFs are even more resistant to FAD-induced cell death $(P<0.01$, Figure $5 d$ ). This resistance is correlated with significantly impaired ER stress responses as shown by a lack of CHOP induction (Figure 5f) and significantly decreased GRP78 accumulation and XBP1 splicing (Figures $5 e$ and f). XBP1 targets in UPR signaling include genes that help to reestablish ER homeostasis and promote survival as well as genes such as CHOP that promote cell death. ${ }^{5}$ However, it is not necessarily expected that XBP1 ${ }^{-1-} \mathrm{MEFs}$ will exhibit more resistance to FAD-induced cell death than PERK ${ }^{-1-}$ MEFs. Interestingly, XBP1 ${ }^{-1-}$ MEFs show increased basal levels of GRP78 protein and XBP1 splicing as compared with control or PERK ${ }^{-1-}$ MEFs (compare panels $e$ and $f$ to $b$ and $\mathrm{C}$ in Figure 5), indicating that the XBP1 ${ }^{-1}$ MEFs have an increased basal level of ER stress, which potentially contributes to decreased induction of ER stress and cell death by FAD. Taken together, the results from PERK ${ }^{-1-}$ and XBP1 ${ }^{-1-}$ MEFs provide further support that cell death induced by $F A D$ is mediated by ER stress.

FAD inhibits tumor growth in a xenograft model of human colorectal cancer. As there has been limited in vivo evidence that FAD can suppress colorectal cancer growth, we investigated the anticancer activity of FAD using a xenograft model of HCT116 human colorectal cancer cells. Exponentially growing firefly luciferase-tagged HCT116 cells were inoculated into the flanks of athymic nude mice. Beginning on day 1 , animals were also administered with FAD or vehicle at 10 or $15 \mathrm{mg} / \mathrm{kg} /$ day. Tumor growth was measured by Xenogeny bioluminescence imaging at the indicated time points. As shown in Figure $6 \mathrm{a}$, treatment with FAD significantly decreases the Xenogen (Caliper Life Sciences, Hopkinton, MA, USA) imaging signal. Quantitative analysis of the imaging data (Figure 6b) reveals that treatment with $15 \mathrm{mg} / \mathrm{kg}$ of FAD significantly inhibits xenograft tumor growth from day $19(P<0.05$ versus control). Further treatment with either 10 or $15 \mathrm{mg} / \mathrm{kg}$ of FAD causes significantly decreased Xenogen imaging signals compared with control at the end point, day $28(P<0.01)$. These results demonstrate that FAD can inhibit human tumor growth in a xenograft model.

As most chemotherapeutic agents are delivered in combination when cures are achieved, we tested whether FAD can be used in combination with currently approved therapeutic agents to enhance cancer cell killing and/or reduce toxicity to normal cells. 5-FU is a chemotherapeutic drug that has been approved for use in treating colorectal cancer. In the presence of $3 \mu \mathrm{M}$ of $\mathrm{FAD}, 5-\mathrm{FU}$ exhibits a much stronger cytotoxic effect on HCT116 cells even at much lower concentrations (Figure 6c). These data indicate that FAD has a synergistic effect with 5-FU in killing colorectal cancer cells and can potentially be used to increase the effectiveness and/or lower the side effects of 5-FU in cancer therapies.

\section{Discussion}

FAD is a bioactive compound isolated from Oplopanax horridus, a plant used extensively by indigenous people to heal a variety of ailments. ${ }^{30}$ We show here that FAD preferentially kills cancer cells and significantly inhibits tumor growth. Furthermore, FAD significantly increases the cancerkilling activity of 5-FU, an approved chemotherapeutic drug used for treating advanced colorectal cancers. Taken together, these results suggest that FAD can potentially be 
a

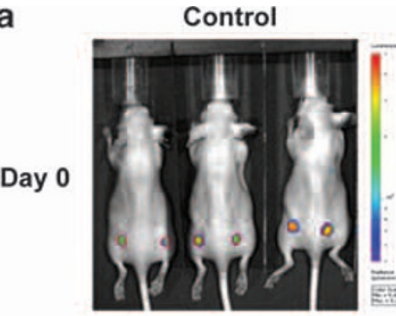

FAD $10 \mathrm{mg} / \mathrm{kg}$
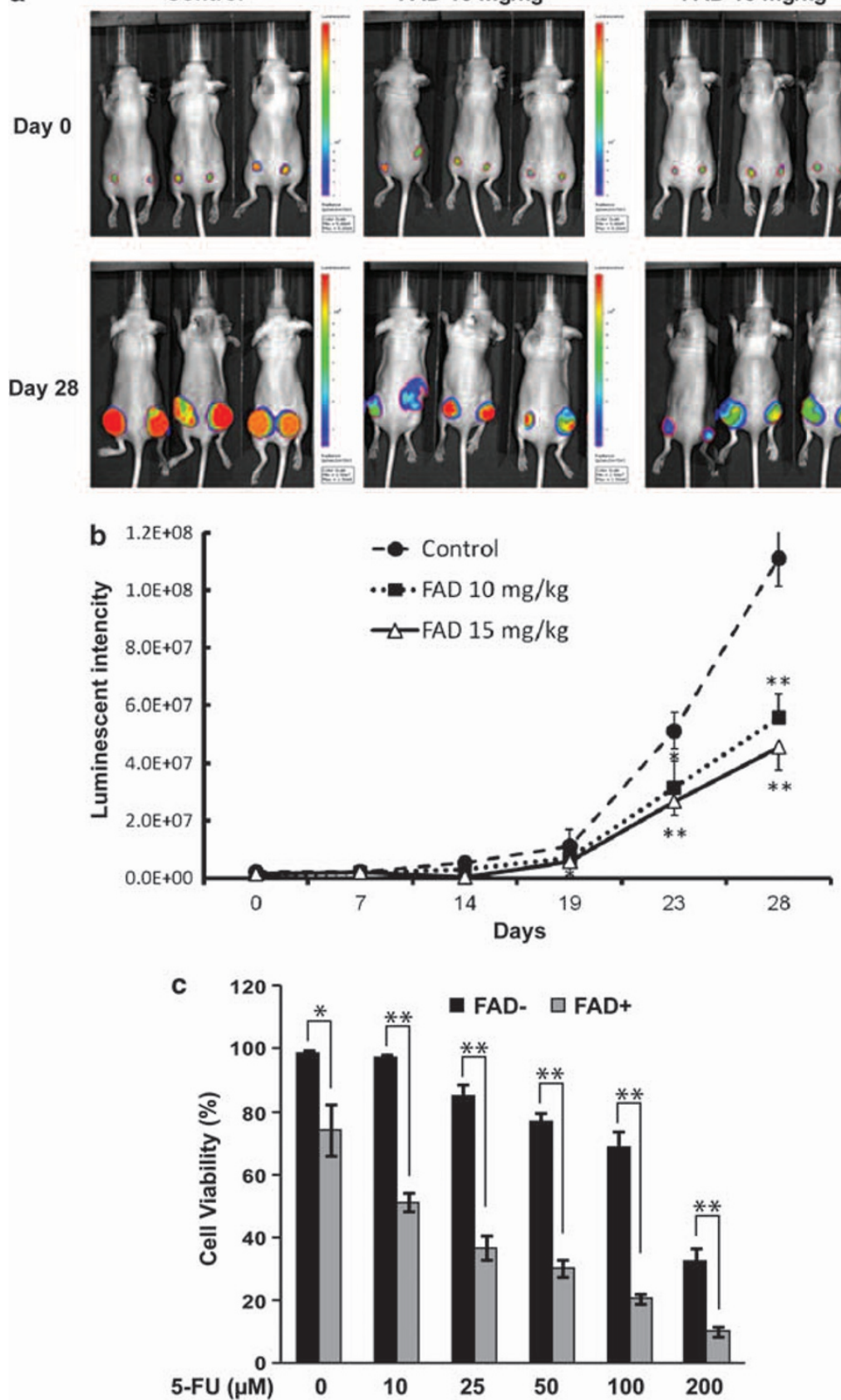

FAD $15 \mathrm{mg} / \mathrm{kg}$
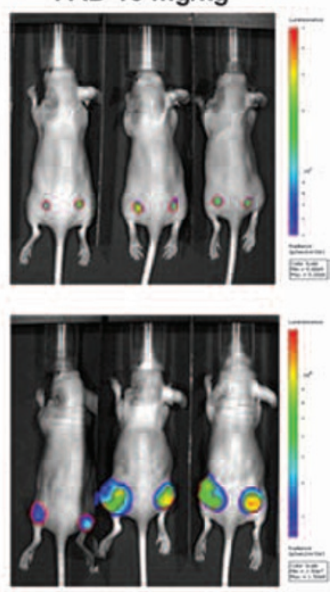
Terminally misfolded proteins in the ER are removed by ERAD. Ubiquitination of ERAD substrates is required not only for subsequent dislocation from the ER membrane, but also for targeting dislocated polypeptides to the $26 \mathrm{~S}$ proteasome for degradation. ${ }^{32}$ Indeed several studies have suggested that the proteasome inhibitor bortezomib (Velcade), the first proteasome inhibitor clinically approved for treating multiple myeloma and mantle cell lymphoma, ${ }^{33,34}$ induces ER stress through interfering with ERAD, which contributes to its cytotoxic activity against cancer cells. ${ }^{27,28}$ Therefore, although it is traditionally thought that the stabilization of a particular subset of proteins might be responsible for the antitumorigenic activity of bortezomib, it is equally likely that its efficacy results from enhanced proteotoxic stress. ${ }^{4}$

Cancer cells generally exhibit increased cellular stresses and are more dependent on stress support pathways for survival. This makes cancer cells more susceptible to either stress sensitization or stress overload. ${ }^{4}$ Indeed stress overload and stress sensitization are likely the mechanism behind a large number of chemotherapeutic agents currently in use, such as DNA damaging agents, inhibitors of DNA replication, inhibitors of mitosis, and proteasome inhibitors. Furthermore, agents that target distinct branches of cellular stress responses can potentially act synergistically in inducing stress overload in cancer cells, leading to enhanced cancer cell killing or reduced side effects. Consistent with this, we find that FAD and 5-FU, which induce ER stress and DNA replication/ damage stress respectively, show synergistic effects in killing cancer cells. It is quite possible that FAD can also induce synergistic cancer cell killing with other chemotherapeutic agents that induce distinct cellular stresses on cancer cells.

\section{Materials and Methods \\ Plant materials and phytochemical isolation of FAD. The root bark of Oplopanax horridus (Sm.) Miq. was obtained from Pacific Botanicals, LLC and was authenticated by a botanist. The dried root bark was extracted with $80 \%$ ethanol, and the crude extract was suspended in water. After washing with petroleum ether (b.p. $60-90^{\circ} \mathrm{C}$ ), the water phase was extracted with ethyl acetate. The ethyl acetate fraction was separated by silica gel and RP- $\mathrm{C}_{18}$ silica gel to afford FAD. The purity was $>98 \%$ by HPLC analysis. ${ }^{35}$}

Chemicals and reagents. Z-VAD-fmk and Necrostatin-1 were obtained from Enzo Life Sciences (Farmingdale, NY, USA). MG132 was obtained from Fisher Scientific (Chicago, IL, USA). 5-FU was obtained from Acros Organics (Fair Lawn, NJ, USA). Annexin V apoptosis kit was obtained from BD Biosciences (San Jose, CA, USA). Anti-GRP78, anti-LC3 and anti-PERK antibodies were obtained from Cell Signaling Technology (Danvers, MA, USA). Anti-Ubiquitin and anti- $\beta$-actin antibodies were obtained from Santa Cruz Biotechnology (Santa Cruz, CA, USA).

Cell culture. Normal human colon epithelial cells, FHC, and human colorectal cancer cells, HCT116 and SW480, were obtained from ATCC (Manassas, VA, USA). MEFs used were established by SV40 large T antigen and were described previously. ${ }^{36,37} \mathrm{FHC}$, MCF10A, and IEC6 cells were maintained in the ATCCsuggested complete growth medium. HCT116, SW480, MDA-MB231, MCF7, and DU145 were maintained in DMEM medium supplemented with $10 \%$ fetal bovine serum, RAJI and KOPN-1 were maintained in RPMI medium supplemented with $10 \%$ fetal bovine serum, MonoMac6 were maintained in RPMl medium supplemented with $10 \%$ fetal bovine serum, $2 \mathrm{mM} \mathrm{L-glutamine,} 1 \%$ nonessential amino acid, $1 \mathrm{mM}$ sodium pyruvate, and $9 \mu \mathrm{g} / \mathrm{ml}$ human insulin. All cells cultured in a humidified chamber with $5 \% \mathrm{CO}_{2}$ at $37^{\circ} \mathrm{C}$.

Trypan blue staining, FACS analysis and western blot analysis. Cells were collected and stained with $0.4 \%$ Trypan Blue dye in PBS for $5 \mathrm{~min}$ at room temperature. Cell death was determined as the percent of cells that incorporated dye. At least three independent experiments were carried out to obtain the means and S.D's. Student's two-tailed $t$-test was used to determine statistical significance. FACS analysis and western blot analysis was carried out as described by Li et al. ${ }^{38}$

RNA isolation and RT-PCR. Total RNA was extracted with the RNeasy Mini Kit according to the manufacturer's instructions (Qiagen, Valencia, CA, USA). cDNA was synthesized using M-MLV reverse transcriptase from Promega (Madison, WI, USA). Primer pairs used for RT-PCR are: human XBP1, 5'-GGAGT TAAGACAGCGCTTGG-3' (sense) and 5'-ACTGGGTCCAAGTTGTCCAG-3' (antisense); human CHOP, $5^{\prime}$-TGGAAGCCTGGTATGAGGAC-3' (sense) and $5^{\prime}$-TGT GACCTCTGCTGGTTCTG-3' (antisense); human GRP78, 5'-TGAAGAGCTCAAC ATGGATCTGTT-3' (sense) and 5'-CTACAGCTTCATCTGGGTTTATGC-3' (antisense); human GAPDH, $5^{\prime}$-CTCTGACTTCAACAGCGACAC-3' (sense) and $5^{\prime}$-CA TACCAGGAAATGAGCTTGACAA-3' (antisense); mouse XBP1, 5'-ACACGCTTG GGAATGGACAC-3' (sense), Rat XBP1, 5'-GCTTGTGATTGAGAACCAGG-3' (sense), and $5^{\prime}$-CCATGGGAAGATGTTCTGGG-3' (mouse/Rat antisense); mouse CHOP, 5' $5^{\prime}$-CCTAGCTTGGCTGACAGAGG-3' (sense) and 5'-CTGCTCCTTCTCCT TCATGC-3' (antisense); mouse/Rat GAPDH, $5^{\prime}$-GCACAGTCAAGGCCGAGAAT$3^{\prime}$ (sense) and 5'-GCCTTCTCCATGGTGGTGAA-3' (antisense).

Plasmids and lentiviral preparation and transduction. Human GRP78 cDNA was subcloned into the lentiviral expression vector pCDH-CMVEF1-puro (System Biosciences, Mountain View, CA, USA). The pLKO.1 lentiviral shRNA expression system was used to generate 2 different sh-GRP78 constructs (sh-GRP78-1, 5' -CTTGTTGGTGGCTCGACTCGA-3') and (sh-GRP78-2, 5'-GCCT AAATGTTATGAGGATCATT- $3^{\prime}$ ). Viral packaging was done according to the protocol described by Li et al. ${ }^{39}$

Xenograft tumor model of human colon cancer and Xenogen bioluminescence imaging. A human colorectal cancer cell line, HCT116Luc, was used to establish a xenograft model in immunodeficient female BALB/C nude mice (4-6 weeks of age, Harlan, Indianapolis, IN, USA). In all, $1 \times 10^{6}$ HCT116-Luc cells in $50 \mu$ PBS were injected into both flanks of each animal. FAD was administered intraperitoneally starting the same day at doses of 10 or $15 \mathrm{mg} /$ $\mathrm{kg}$ every day. Control mice were injected with FAD vehicle. Animal whole-body optical imaging was carried out with the Xenogen IVIS 200 imaging system as previously described by $\mathrm{He}$ et al. ${ }^{40}$

Statistical analysis. Values are presented as mean \pm S.D. Statistical significance was assessed by Student's two-tailed $t$-test and $P<0.05$ was considered statistically significant.

\section{Conflict of Interest}

The authors declare no conflict of interest.

Acknowledgements. We thank Drs David Ron and Laurie Glimcher for the $\mathrm{PERK}^{-1-}$ and XBP1 ${ }^{-1-}$ MEFs, Dr. Jianjun Chen for the RAJl, MonoMac6, and KOPN-1 cells, Dr. Jinhua Xu for the MCF10A cells, Dr. Geoffrey Greene for the MDA-MB231 and MCF7 cells. We also thank Dr. Gopal Thinakaran and Gabe Gordon for reading the manuscript. This work was supported in part by the following grants: NIH/NCCAM AT004418, NIH GM074197, NIH/NCl CA149275, DOD W81XWH-10-1-0077, and UL015A from the University of Macau.

1. Siegel R, Naishadham D, Jemal A. Cancer statistics, 2012. CA Cancer J Clin 2012; 62: 10-29.

2. Schnell FM. Chemotherapy-induced nausea and vomiting: the importance of acute antiemetic control. Oncologist 2003; 8: 187-198.

3. Wang CZ, Basila D, Aung HH, Mehendale SR, Chang WT, McEntee E et al. Effects of ganoderma lucidum extract on chemotherapy-induced nausea and vomiting in a rat model. Am J Chin Med 2005; 33: 807-815.

4. Luo J, Solimini NL, Elledge SJ. Principles of cancer therapy: oncogene and non-oncogene addiction. Cell 2009; 136: 823-837.

5. Kim I, Xu W, Reed JC. Cell death and endoplasmic reticulum stress: disease relevance and therapeutic opportunities. Nat Rev Drug Discov 2008; 7: 1013-1030.

6. Walter $P$, Ron $D$. The unfolded protein response: from stress pathway to homeostatic regulation. Science 2011; 334: 1081-1086. 
7. Lee AS. GRP78 induction in cancer: therapeutic and prognostic implications. Cancer Res 2007; 67: 3496-3499.

8. Vembar SS, Brodsky JL. One step at a time: endoplasmic reticulum-associated degradation. Nat Rev Mol Cell Biol 2008; 9: 944-957.

9. Tabas I, Ron D. Integrating the mechanisms of apoptosis induced by endoplasmic reticulum stress. Nat Cell Biol 2011; 13: 184-190.

10. Thomenius MJ, Distelhorst CW. Bcl-2 on the endoplasmic reticulum: protecting the mitochondria from a distance. J Cell Sci 2003; 116(Part 22): 4493-4499.

11. Egger L, Schneider J, Rheme C, Tapernoux M, Hacki J, Borner C. Serine proteases mediate apoptosis-like cell death and phagocytosis under caspase-inhibiting conditions. Cell Death Differ 2003; 10: 1188-1203.

12. Metzger BT, Barnes DM, Reed JD. Purple carrot (Daucus carota L.) polyacetylenes decrease lipopolysaccharide-induced expression of inflammatory proteins in macrophage and endothelial cells. J Agric Food Chem 2008; 56: 3554-3560.

13. Zschocke S, Lehner M, Bauer R. 5-Lipoxygenase and cyclooxygenase inhibitory active constituents from Qianghuo (Notopterygium incisum). Planta Med 1997; 63: 203-206.

14. Deng S, Wang Y, Inui T, Chen SN, Farnsworth NR, Cho S et al. Anti-TB polyynes from the roots of Angelica sinensis. Phytother Res 2008; 22: 878-882.

15. Stavri M, Gibbons S. The antimycobacterial constituents of dill (Anethum graveolens) Phytother Res 2005; 19: 938-941.

16. Sun S, Du GJ, Qi LW, Williams S, Wang CZ, Yuan CS. Hydrophobic constituents and their potential anticancer activities from Devil's Club (Oplopanax horridus Miq.) $J$ Ethnopharmacol 2010; 132: 280-285.

17. Ohnuma T, Anan E, Hoashi R, Takeda Y, Nishiyama T, Ogura K et al. Dietary diacetylene falcarindiol induces phase 2 drug-metabolizing enzymes and blocks carbon tetrachlorideinduced hepatotoxicity in mice through suppression of lipid peroxidation. Biol Pharm Bull 2011; 34: 371-378.

18. Degterev A, Hitomi J, Germscheid M, Ch'en IL, Korkina O, Teng X et al. Identification of RIP1 kinase as a specific cellular target of necrostatins. Nat Chem Biol 2008; 4 : 313-321.

19. Tasdemir E, Galluzzi L, Maiuri MC, Criollo A, Vitale I, Hangen E et al. Methods for assessing autophagy and autophagic cell death. Methods Mol Biol 2008; 445: 29-76.

20. Yorimitsu T, Nair U, Yang Z, Klionsky DJ. Endoplasmic reticulum stress triggers autophagy. J Biol Chem 2006; 281: 30299-30304.

21. Shi YH, Ding ZB, Zhou J, Hui B, Shi GM, Ke AW et al. Targeting autophagy enhances sorafenib lethality for hepatocellular carcinoma via ER stress-related apoptosis. Autophagy 2011; 7: 1159-1172.

22. Xu Y, Yu H, Qin H, Kang J, Yu C, Zhong J et al. Inhibition of autophagy enhances cisplatin cytotoxicity through endoplasmic reticulum stress in human cervical cancer cells. Cancer Lett 2012; 314: 232-243.

23. Pyrko $\mathrm{P}$, Schonthal AH, Hofman FM, Chen TC, Lee AS. The unfolded protein response regulator GRP78/BiP as a novel target for increasing chemosensitivity in malignant gliomas. Cancer Res 2007; 67: 9809-9816.

24. Virrey JJ, Dong D, Stiles C, Patterson JB, Pen L, Ni M et al. Stress chaperone GRP78/BiP confers chemoresistance to tumor-associated endothelial cells. Mol Cancer Res 2008; 6: $1268-1275$.
25. Harding HP, Zhang $\mathrm{Y}$, Bertolotti $\mathrm{A}$, Zeng $\mathrm{H}$, Ron D. Perk is essential for translational regulation and cell survival during the unfolded protein response. Mol Cell 2000; 5: 897-904.

26. Little JL, Wheeler FB, Fels DR, Koumenis C, Kridel SJ. Inhibition of fatty acid synthase induces endoplasmic reticulum stress in tumor cells. Cancer Res 2007; 67: 1262-1269.

27. Fribley $A$, Zeng $Q$, Wang $C Y$. Proteasome inhibitor PS-341 induces apoptosis through induction of endoplasmic reticulum stress-reactive oxygen species in head and neck squamous cell carcinoma cells. Mol Cell Biol 2004; 24: 9695-9704.

28. Obeng EA, Carlson LM, Gutman DM, Harrington WJ Jr, Lee KP, Boise LH. Proteasome inhibitors induce a terminal unfolded protein response in multiple myeloma cells. Blood 2006; 107: 4907-4916.

29. Gregory MA, Hann SR. c-Myc proteolysis by the ubiquitin-proteasome pathway: stabilization of c-Myc in Burkitt's lymphoma cells. Mol Cell Biol 2000; 20: 2423-2435.

30. Calway T, Du GJ, Wang CZ, Huang WH, Zhao J, Li SP et al. Chemical and pharmacological studies of Oplopanax horridus, a North American botanical. J Nat Med 2012; 66: 249-256.

31. Hasnain SZ, Lourie R, Das I, Chen AC, McGuckin MA. The interplay between endoplasmic reticulum stress and inflammation. Immunol Cell Biol 2012; 90: 260-270.

32. Liu Y, Ye Y. Proteostasis regulation at the endoplasmic reticulum: a new perturbation site for targeted cancer therapy. Cell Res 2011; 21: 867-883.

33. Chauhan D, Hideshima T, Anderson KC. Proteasome inhibition in multiple myeloma: therapeutic implication. Annu Rev Pharmacol Toxicol 2005; 45: 465-476.

34. Adams J, Kauffman M. Development of the proteasome inhibitor Velcade (Bortezomib). Cancer Invest 2004; 22: 304-311.

35. Huang WH, Zhang QW, Wang CZ, Yuan CS, Li SP. Isolation and identification of two new polyynes from a North American ethnic medicinal plant-Oplopanax horridus (Smith) Miq. Molecules 2010; 15: 1089-1096.

36. Harding HP, Zhang Y, Ron D. Protein translation and folding are coupled by an endoplasmic-reticulum-resident kinase. Nature 1999; 397: 271-274.

37. Ito D, Walker JR, Thompson CS, Moroz I, Lin W, Veselits ML et al. Characterization of stanniocalcin 2, a novel target of the mammalian unfolded protein response with cytoprotective properties. Mol Cell Biol 2004; 24: 9456-9469.

38. Li B, Zhao J, Wang CZ, Searle J, He TC, Yuan CS et al. Ginsenoside Rh2 induces apoptosis and paraptosis-like cell death in colorectal cancer cells through activation of p53. Cancer Lett 2011; 301: 185-192.

39. Li B, Gordon GM, Du CH, Xu J, Du W. Specific killing of Rb mutant cancer cells by inactivating TSC2. Cancer Cell 2010; 17: 469-480.

40. He BC, Gao JL, Zhang BQ, Luo Q, Shi Q, Kim SH et al. Tetrandrine inhibits Wnt/betacatenin signaling and suppresses tumor growth of human colorectal cancer. Mol Pharmacol 2011; 79: 211-219.

Cell Death and Disease is an open-access journal published by Nature Publishing Group. This work is licensed under the Creative Commons Attribution-NonCommercial-No Derivative Works 3.0 Unported License. To view a copy of this license, visit http://creativecommons.org/licenses/by-nc-nd/3.0/

Supplementary Information accompanies the paper on Cell Death and Disease website (http://www.nature.com/cddis) 